\title{
Alteraciones de Número en Dentición de Pacientes en- tre 2 y 12 Años de Edad con Disrafias Labio Alvéolo Palatina Atendidos en la Unidad de Odontopediatría del Hospital Regional Antofagasta, Chile
}

\author{
Dentition Number Alterations in Patients between 2 and 12 Years of Age with Cleft Alveolus \\ Palate Examined at the Pediatric Dental Unit of the Regional Hospital Antofagasta, Chile
}

Irene Gutiérrez Guerra* \& Oriana Valenzuela Rivera**

GUTIÉRREZ, G. I. \& VALENZUELA, R. O. Alteraciones de número en dentición de pacientes entre 2 y 12 años de edad con disrafias labio alvéolo palatina atendidos en la Unidad de Odontopediatria del Hospital Regional Antofagasta, Chile. Int. J. Odontostomat., 8(3):481-490, 2014.

RESUMEN: Se realizó un estudio de las alteraciones de número dientes en 71 pacientes entre 2 y 12 años de edad, portadores de Fisura Labio Alvéolo Palatina del Hospital Regional de Antofagasta, atendidos entre Abril del 2004 y Julio del 2010, utilizando las fichas clínicas, radiografías panorámicas, oclusales o periapicales. Se encontró una prevalencia de agenesias dentarias en un $57,75 \%$, de dientes supernumerarios en un $23,95 \%$. Sólo el $18,4 \%$ de los niños no presentó alteraciones de número. En la distribución por sexo, en los niños la fisura labial y fisura labio alvéolo palatina bilateral presentó una mayor frecuencia, mientras que en las niñas las fisuras palatinas y fisuras labio alvéolo palatinas unilaterales fueron más frecuentes. Según el tipo de fisura, la más frecuente fue la fisura labio alvéolo palatina unilateral con un $64,79 \%$. La frecuencia de agenesias en el lado de la fisura fue de un $89,3 \%$. El diente más afectado en las agenesias fue el incisivo lateral con un 78,6\% en los niños entre 2 a 6 años, y con un 100\% en los niños entre 7 y 12 años. Se observó que el $61,5 \%$ de los niños con agenesia tenían 1 diente afectado, el 30,8\% mostraron 2 dientes afectados y solo el 7,7\% presentó 3 o más dientes afectados.

PALABRAS CLAVE: fisuras, agenesias, dientes supernumerarios.

\section{INTRODUCCIÓN}

La Fisura Labio Alvéolo Palatina (FLAP), es una de las malformaciones congénitas más frecuentes y se produce por una alteración en la fusión de los tejidos que darán origen al labio superior y al paladar durante el desarrollo embrionario.

Las fisuras representan una condición genética, determinada por la presencia de varios genes y un gen mayor. Su frecuencia mundial es de 1 por cada 1.200 nacidos vivos. En Chile afecta casi al doble de la tasa mundial, 1,8 por cada 1.000 nacidos vivos aproximadamente. En cuanto a su distribución geográfica, el $62 \%$ de ellos se distribuye en las regiones Metropolitana, V y VIII. En la Segunda Región de nuestro país se encuentra la más alta incidencia a nivel nacional con 1 por 259 nacidos vivos (MINSAL, 2009).
Las fisuras labio alvéolo palatinas originan problemas en las estructuras óseas del maxilar, y originana así una alteración en el perfil facial; frecuentemente encontramos comunicaciones oronasales que ocasionan problemas en la deglución, respiración y fonética del paciente. El desarrollo del proceso alveolar en la región del paladar de estos pacientes determina una serie de anomalías dentarias tanto en su número y forma, lo que constituye un factor etiológico de maloclusiones que se encuentran en la totalidad de los pacientes portadores de fisuras, ocasionando problemas funcionales y estéticos (MINSAL).

Basados en datos del MINSAL, que indican que la Región de Antofagasta presenta una alta tasa de incidencia de fisuras alvéolo palatinas, se realizó este

\footnotetext{
* Unidad de Fisurados - Odontopediatría, Hospital Regional de Antofagasta, Antofagasta, Chile.

"Facultad de Medicina y Odontología, Universidad de Antofagasta, Antofagasta, Chile.
} 
trabajo de investigación con el propósito de describir las alteraciones de número en dentición de niños entre 2-12 años de edad portadores de fisura labio alveolo palatina atendidos en la Unidad de Fisurados Odontopediatría del Servicio Dental del Hospital Regional de Antofagasta, en el periodo comprendido entre abril del 2004 a julio del 2010, identificando conjuntamente los tipos de fisura y alteración de número según sexo de los pacientes, identificando además las alteraciones de número según tipo de fisura, número y diente afectado, estableciendo también la ubicación de las alteraciones de número según el lado de la fisura.

\section{MATERIAL Y METODO}

Se realizó un estudio descriptivo de corte transversal, para evaluar las alteraciones de número que presentan los pacientes con disrafias labio alvéolo palatinas. Se revisaron 91 historias clínicas de pacientes que fueron atendidos por fisura labio alvéolo palatina en la Unidad de Fisurados - Odontopediatría del Hospital Regional de Antofagasta, en el periodo de abril del año 2004 a julio del año 2010; de éste universo, 71 pacientes constituyeron la muestra que cumplía con los criterios de inclusión. Se incluyeron pacientes con diagnóstico de fisura labio alvéolo palatina, sea esta unilateral o bilateral y hayan sido atendidos en la Unidad de Fisurados - Odontopediatría del Hospital Regional de Antofagasta entre abril del 2004 a julio del 2010, con edades entre 2 y 12 años, todos ellos con sus respectivas historias clínicas completas, que tuvieran radiografías panorámicas, oclusales o periapicales y que fueron controlados en la Unidad de Fisurados - Odontopediatría durante el año 2010. Fueron excluidos pacientes que no fueron ubicados en forma telefónica, por Oficina de Partes del Hospital Regional de Antofagasta o vía correo postal. El estudio contó con la autorización del Director del Hospital Regional de Antofagasta.

La totalidad de los pacientes contaba con radiografía panorámica, oclusal o periapical. A cada paciente se le realizó un examen clínico apoyado con las imágenes radiográficas. Se recolectaron datos sobre sexo, edad, tipo de fisura, alteraciones de número como agenesias y supernumerarios. Se tomó como referencia la edad del niño en el momento en que se realizó la radiografía panorámica.

Se realizó una evaluación de las radiografías, enfatizándose en los siguientes aspectos: número total de dientes en la radiografía panorámica, número de dientes ausentes, presencia de dientes supernumerarios. Dentro de los criterios diagnósticos se consideró como lado de la fisura, al área distal adyacente a la fisura; mientras que el área mesial fue considerado como el lado opuesto de la fisura, se consideró ausencia congénita de un diente, si es que el germen dentario se encontraba ausente en la radiografía, esto fue apoyado por la edad del niño y en algunos casos la presencia de la diente temporal. Cuando se encontró un diente (incisivo lateral maxilar) en el lado mesial de la fisura y otro diente de las mismas características en el lado distal, se consideró a este último como diente supernumerario.

\section{RESULTADOS}

Se observó que en el grupo entre 2 y 6 años el promedio de edad fue de 3,8 años y en el grupo de pacientes entre 7 y 12 años el promedio fue de 8,6 años.

Del total de la muestra (71 pacientes) el $73,2 \%$ presentaba edades entre 2 a 6 años y el $26,8 \%$ entre 7 a 12 años (Tabla I).

Tabla I. Distribución de pacientes en la muestra estudiada según edad y sexo.

\begin{tabular}{|c|c|c|c|c|c|c|}
\hline \multirow{3}{*}{ Grupo etár eo } & \multicolumn{4}{|c|}{ Sexo } & \multirow{2}{*}{\multicolumn{2}{|c|}{ Total }} \\
\hline & \multicolumn{2}{|c|}{ Masculino } & \multicolumn{2}{|c|}{ Femenino } & & \\
\hline & $\mathrm{n}$ & $\%$ & $\mathrm{n}$ & $\%$ & $\mathrm{n}$ & $\%$ \\
\hline$-6 a$ & 26 & 70,3 & 26 & 76,5 & 52 & 73,2 \\
\hline 7-12 años & 11 & 29,7 & 8 & 23,5 & 19 & 26,8 \\
\hline Total & 37 & 100 & 34 & 100 & 71 & 100 \\
\hline
\end{tabular}

En relación a la distribución de pacientes según tipo de fisura y sexo, se observó que la Fisura Labio palatina unilateral se presentaba en mayor porcentaje tanto en mujeres como hombres, de un total de 37 varones y 34 mujeres, $21(45,6 \%)$ y $25(54,4 \%)$ respectivamente, presentaban este tipo de fisura (Tabla II).

Tabla II. Distribución de pacientes según tipo de fisura y sexo.

\begin{tabular}{lcccccc}
\hline \multirow{2}{*}{ Tipo de fisura } & \multicolumn{4}{c}{ Sexo } & \multicolumn{2}{c}{ Total } \\
\cline { 2 - 7 } & \multicolumn{2}{c}{ Masculino } & \multicolumn{2}{c}{ Femenino } & \multicolumn{2}{c}{} \\
\cline { 2 - 7 } & $\mathrm{n}$ & $\%$ & $\mathrm{n}$ & $\%$ & $\mathrm{n}$ & $\%$ \\
\hline Labial & 4 & 80 & 1 & 20 & 5 & 100 \\
Palatina & 1 & 14,3 & 6 & 85,7 & 7 & 100 \\
Labio Palatina U & 21 & 45,6 & 25 & 54,4 & 46 & 100 \\
Labio Palatina B & 11 & 84,6 & 2 & 15,4 & 13 & 100 \\
Total & 37 & 52,1 & 34 & 47,9 & 71 & 100 \\
\hline
\end{tabular}


El $80 \%$ de los pacientes con fisura labial (Fig. 1A) correspondieron al sexo masculino, sin embargo, en los pacientes con fisura palatina (Fig. 1B), el $85,7 \%$ correspondió al sexo femenino, la fisura labio palatina unilateral (Fig. 1C), mostró una frecuen- cia similar entre niñas y niños, siendo un poco mayor en las niñas con el $54,4 \%$ contra el $45,6 \%$ los niños. Así mismo, la fisura labio palatina bilateral (Fig. 1D) fue más frecuente en el sexo masculino con el $84,6 \%$.

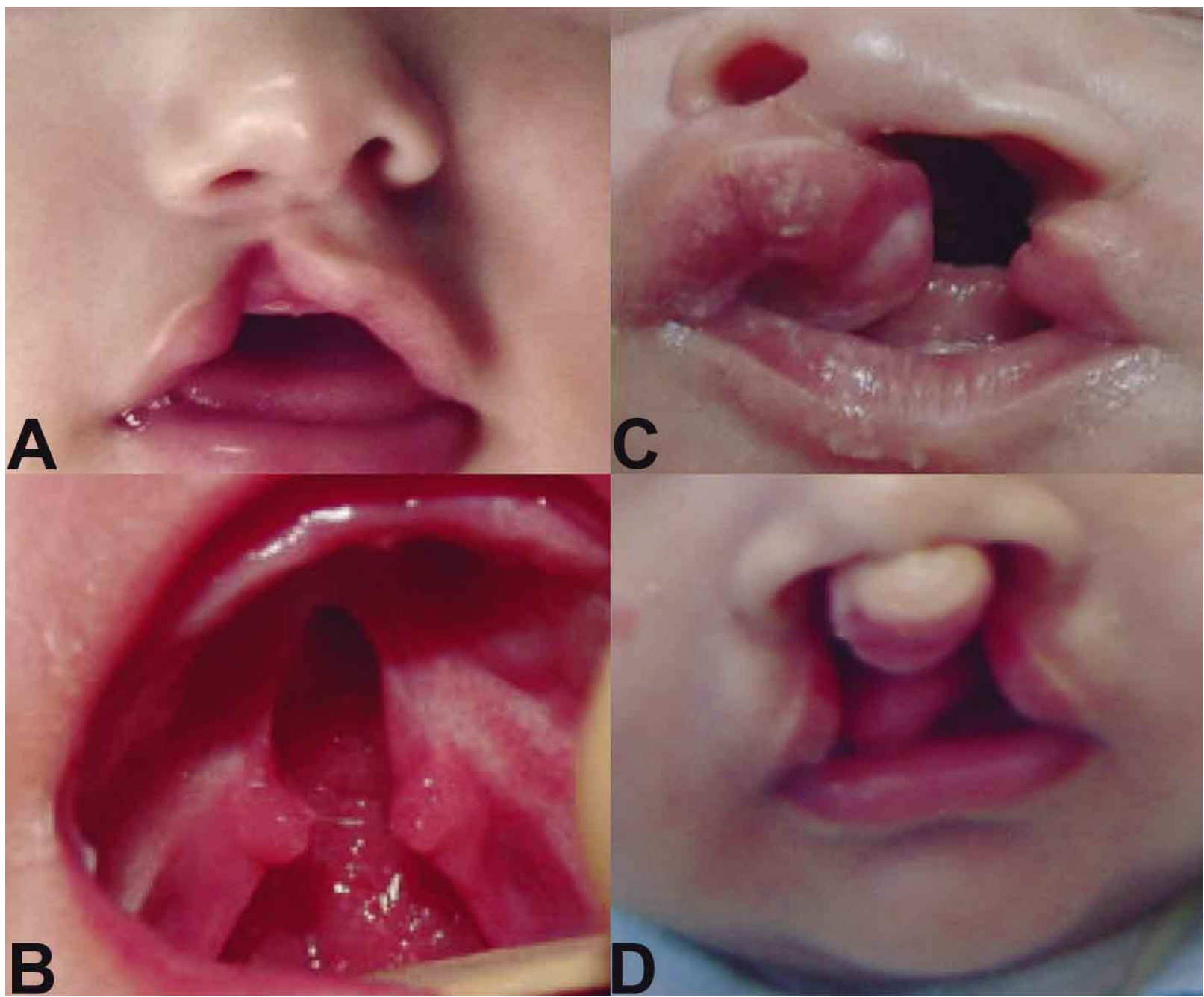

Fig. 1. Tipos de fisuras encontradas en la población estudiada. A. Fisura Labial. B. Fisura Palatina. C. Fisura Labio Palatina Unilateral. D. Fisura Labio Palatina Bilateral.

De acuerdo a la distribución de pacientes según tipo de fisura y edad, se identificó que la totalidad de los pacientes con fisura labial y palatina tenían entre 2 y 6 años de edad. La fisura labio palatina unilateral se encontró con mayor frecuencia en los pacientes que tenían entre 2 y 6 años con un $69,5 \%$, muy similar a lo encontrado en los pacientes con fisura labio palatina bilateral con un $61,5 \%$, mientras que en los pacientes con edades entre 7 y 12 años la frecuencia fue mucho menor con el $30,5 \%$ y $38,5 \%$ respectivamente (Tabla III).
Tabla III. Distribución de pacientes según tipo de fisura y edad.

\begin{tabular}{lcccccc}
\hline \multirow{2}{*}{ Tipo de fisura } & \multicolumn{4}{c}{ Edad } & \multicolumn{2}{c}{ Total } \\
\cline { 2 - 7 } & \multicolumn{2}{c}{ 2-6 años } & \multicolumn{2}{c}{$7-12$ años } & \multicolumn{1}{c}{} \\
\cline { 2 - 7 } & $\mathrm{n}$ & $\%$ & $\mathrm{n}$ & $\%$ & $\mathrm{n}$ & $\%$ \\
\hline Labial & 5 & 100 & -- & 0 & 5 & 100 \\
Palatina & 7 & 100 & -- & 0 & 7 & 100 \\
Labio Palatina U & 32 & 69,5 & 14 & 30,5 & 46 & 100 \\
Labio Palatina B & 8 & 61,5 & 5 & 38,5 & 13 & 100 \\
Total & 52 & 73,2 & 19 & 26,8 & 71 & 100 \\
\hline
\end{tabular}


Al observar la distribución de pacientes según la alteración de número y sexo, se pudo identificar que la presencia de dientes supernumerarios (Fig. 2) y agenesia dentaria (Figs. 3 y 4 ), se encontraron con mayor frecuencia en el sexo masculino, con un $64,7 \%$ y 53,6

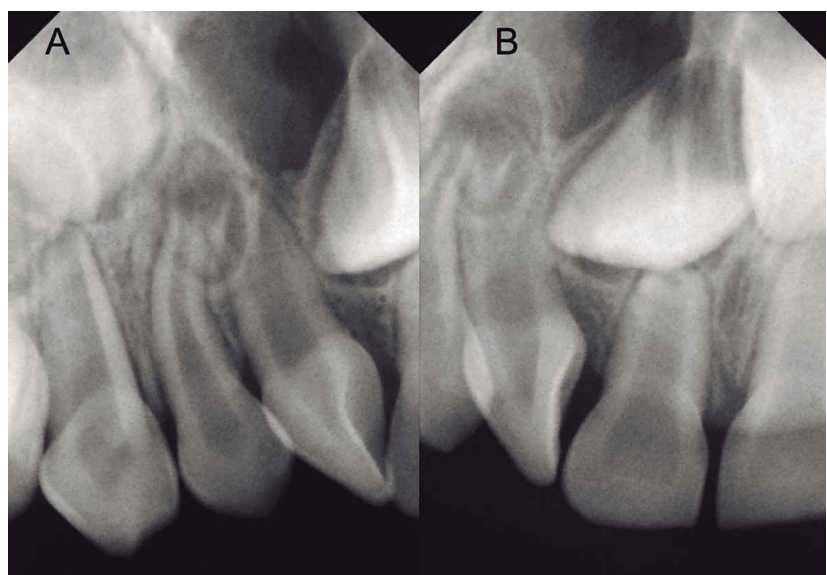

Fig. 2. Paciente sexo masculino. A) Fisura palatina unilateral derecha. Incisivo central decidual derecho supernumerario en giroversión. B) Incisivo lateral definitivo derecho microdóntico. Incisivo central definitivo derecho en giroversión.

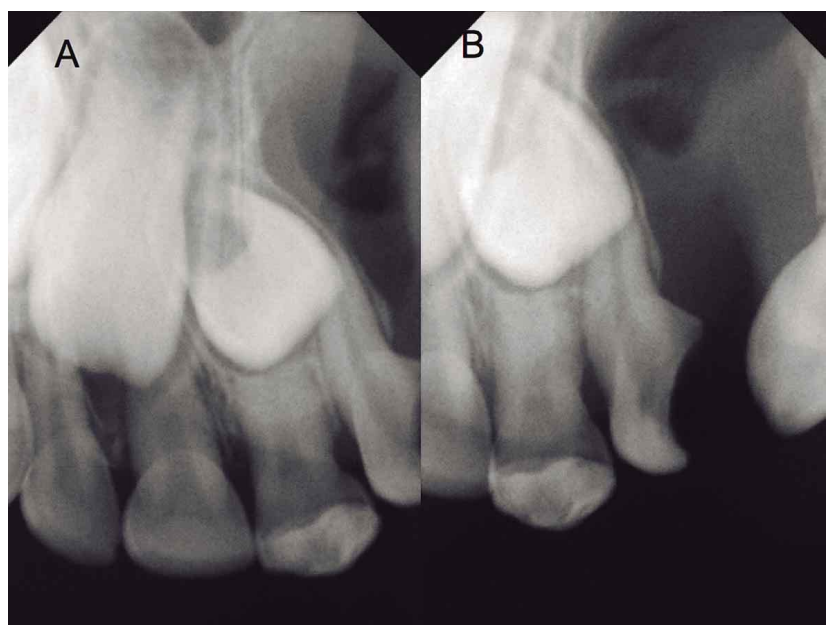

Fig. 3. Paciente sexo femenino. A) Fisura palatina unilateral izquierda. Alteración de cronología de erupción Incisivo central izquierdo. B) Agenesia Incisivo lateral definitivo izquierdo.

Tabla IV. Distribución de pacientes según tipo de anomalía de número y sexo.

\begin{tabular}{lcccccc}
\hline \multirow{2}{*}{ Anomalía de Número } & \multicolumn{9}{c}{ Sexo } & \multicolumn{2}{c}{ Total } \\
\cline { 2 - 8 } & \multicolumn{1}{c}{ Masculino } & \multicolumn{1}{c}{ Femenino } & & \\
\cline { 2 - 8 } & $\mathrm{n}$ & $\%$ & $\mathrm{n}$ & $\%$ & $\mathrm{n}$ & $\%$ \\
\hline Agenesia & 22 & 53,6 & 19 & 46,4 & 41 & 100 \\
Supernumerario & 11 & 64,7 & 6 & 35,3 & 17 & 100 \\
Sin Alteración & 4 & 30,8 & 9 & 69,2 & 13 & 100 \\
Total & 37 & 52,1 & 34 & 47,9 & 71 & 100 \\
\hline
\end{tabular}

$\%$ respectivamente. En los pacientes no afectados por alteraciones de número dentario (Fig. 5), el 69,2\% correspondió al sexo femenino y el $30,8 \%$ al sexo masculino. Los casos de agenesia correspondieron al $46,4 \%$ y de supernumerarios al $35,3 \%$ en las niñas afectadas (Tabla IV).

La distribución de los pacientes según tipo de alteración de número y edad mostrada evidenció que la agenesia y los supernumerarios se presentaron con mayor frecuencia en niños que tenían entre 2 a 6 años de edad con el $68,3 \%$ y $64,7 \%$ afectado, respectivamente. Los niños entre 7 y 12 años mostraron menor frecuencia de estas alteraciones, $31,7 \%$ presentaron agenesia y $35,3 \%$ presencia de supernumerarios (Tabla $\mathrm{V})$. En los pacientes de 2 a 6 años de edad según

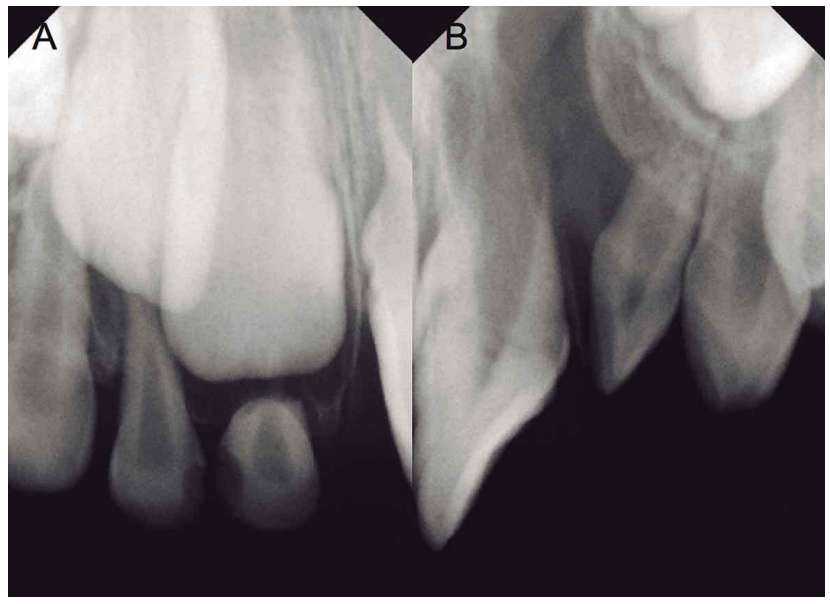

Fig. 4. Paciente sexo masculino. A) Incisivo central definitivo izquierdo erupcionado en giroversión. B) Fisura palatina unilateral izquierda. Agenesia Incisivo lateral definitivo izquierdo.

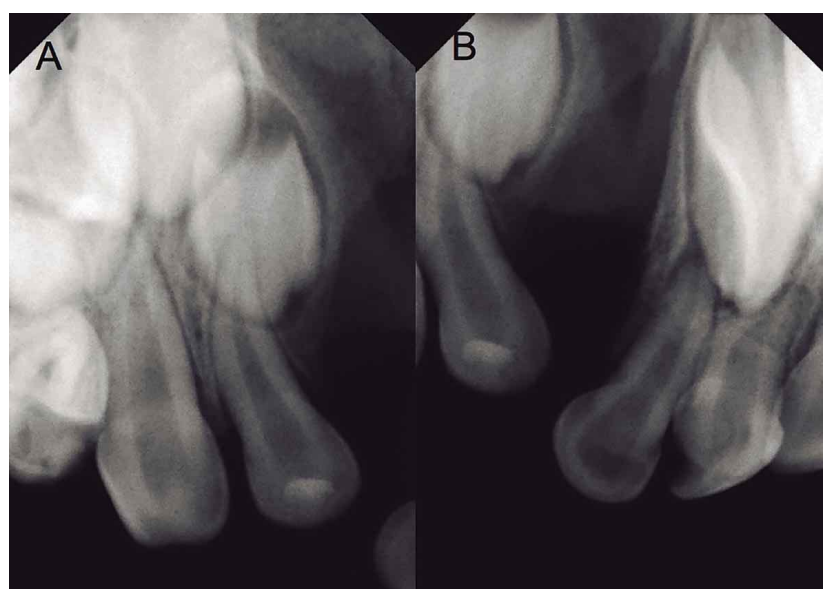

Fig. 5. Paciente sexo femenino. A) Fisura palatina unilateral derecha. Sin alteración de número. Alteración de forma de Incisivo lateral definitivo derecho. B) Incisivo central definitivo derecho en giroversión. 
GUTIÉRREZ, G. I. \& VALENZUELA, R. O. Alteraciones de número en dentición de pacientes entre 2 y 12 años de edad con disrafias labio alvéolo palatina atendidos en la Unidad de Odontopediatria del Hospital Regional Antofagasta, Chile. Int. J. Odontostomat., 8(3):481-490, 2014.

Tabla. V. Distribución de pacientes según tipo de alteración de número y edad.

\begin{tabular}{lcccccc}
\hline \multirow{2}{*}{ Anomalía de Número } & \multicolumn{9}{c}{ Edad } & \multicolumn{2}{c}{ Total } \\
\cline { 2 - 7 } & $2-6$ & años & $7-12$ & años & & \\
\cline { 2 - 7 } & $\mathrm{n}$ & $\%$ & $\mathrm{n}$ & $\%$ & $\mathrm{n}$ & $\%$ \\
\hline Agenesia & 28 & 68,3 & 13 & 31,7 & 41 & 100 \\
Supernumeranio & 11 & 64,7 & 6 & 35,3 & 17 & 100 \\
Sin alteración & 13 & 100 & 0 & 0 & 13 & 100 \\
Total & 52 & 73,2 & 19 & 26,8 & 71 & 100 \\
\hline
\end{tabular}

alteración de número y tipo de fisura, se pudo observar que el $40,0 \%$ de los niños con fisura labial presentaron dientes supernumerarios y un $60,0 \%$ no presentaron alteraciones de número.

Los niños con fisura labial no presentaron agenesia. Por otro lado se pudo determinar además que el $57,1 \%$ de los niños que presentaron fisura palatina tenían agenesia, el $28,6 \%$ supernumerarios y sólo el $14,3 \%$ no tenían alteraciones. Por su parte el $68,7 \%$ de los niños con fisura labio alveolo palatina unilateral mostraron agenesia, el 18,8\% tenían dientes supernumerarios y sólo el $12,5 \%$ no mostraban alteraciones. El $25,0 \%$ de aquellos que tenían fisura labio alvéolo palatina bilateral mostraron agenesia, el $12,5 \%$ tenían dientes supernumerario y un mayor porcentaje $62,5 \%$ no mostraron alteraciones de número (Tabla $\mathrm{VI}$ ).
En la distribución de niños entre 7 a 12 años según alteración de número y tipo de fisura, se pudo observar que estos niños no mostraron fisuras de tipo labial o palatina. El $83,3 \%$ de los niños que mostraron fisura labio palatina unilateral tenían agenesia y el $16,7 \%$ presentaban dientes supernumerarios. Sin embargo los niños de esta edad que presentaban fisura labio palatina bilateral mostraron con mayor frecuencia dientes supernumerarios con un $57,2 \%$ de ellos y en menor frecuencia un $42,8 \%$ mostraron agenesia (Tabla VII).

Al evaluar el número de dientes afectados en los niños de 2 a 6 años según la alteración de número, se encontró que de los 28 niños que mostraron agenesia 19 presentaron 1 solo diente afectado para un $67,8 \%$, seguido del $28,6 \%$ con 2 dientes afectados, solo el 3,6\% tenían 3 o más dientes. De los 11 niños con dientes supernumerarios, 10 presentaron 1 solo diente afectado para un $90,9 \%$ y solo 1 presentaba 2 dientes afectados (Tabla VIII).

La distribución de pacientes de 7 a 12 años según anomalías de número y cantidad de dientes afectados, se pudo observar que el $61,5 \%$ de los niños con agenesia tenían 1 diente afectado, el 30,8\% mostraron 2 dientes afectados y solo el 7,7\% presentó 3 o más dientes afectados. El $83,3 \%$ de los niños que te-

Tabla VI. Distribución de pacientes de 2 a 6 años de edad según alteración de número y tipo de fisura.

\begin{tabular}{lcccccccc}
\hline \multirow{2}{*}{ Tipo de fisura } & \multicolumn{9}{c}{ Alteración de número } \\
& \multicolumn{2}{c}{ Agenesia } & \multicolumn{2}{c}{ Supernumerario } & Sin alteración & \multicolumn{2}{c}{ Total } \\
\cline { 2 - 9 } & $\mathrm{n}$ & $\%$ & $\mathrm{n}$ & $\%$ & $\mathrm{n}$ & $\%$ & $\mathrm{n}$ & $\%$ \\
\hline Labial & 0 & --- & 2 & --- & 3 & --- & 5 & --- \\
Palatina & 4 & 57,1 & 2 & 28,6 & 1 & 14,3 & 7 & 100 \\
Labio Palatina U & 22 & 68,7 & 6 & 18,8 & 4 & 12,5 & 32 & 100 \\
Labio Palatina B & 2 & 25 & 1 & 12,5 & 5 & 62,5 & 8 & 100 \\
Total & 28 & 53,85 & 11 & 21,15 & 13 & 25 & 52 & 100 \\
\hline
\end{tabular}

Tabla VII. Distribución de pacientes de 7 a 12 años según alteración de número y tipo de fisura.

\begin{tabular}{lcccccccc}
\hline & \multicolumn{9}{c}{ Anomalía de número } \\
\multicolumn{1}{c}{ Tipo de Fisura } & \multicolumn{2}{c}{ Agenesia } & \multicolumn{2}{c}{ Supernumerario } & \multicolumn{2}{c}{ Sin alteración } & \multicolumn{2}{c}{ Total } \\
\cline { 2 - 10 } & $\mathrm{n}$ & $\%$ & $\mathrm{n}$ & $\%$ & $\mathrm{n}$ & $\%$ & $\mathrm{n}$ & $\%$ \\
\hline Labial & 0 & 0 & 0 & 0 & 0 & 0 & 0 & 0 \\
Palatina & 0 & 0 & 0 & --- & 0 & 0 & 0 & 0 \\
Labio Palatina U & 10 & 83,3 & 2 & 16,7 & 0 & 0 & 12 & 100 \\
Labio Palatina B & 3 & 42,8 & 4 & 57,2 & 0 & 0 & 7 & 100 \\
Total & 13 & 68,4 & 6 & 31,6 & 0 & 0 & 19 & 100 \\
\hline
\end{tabular}


GUTIÉRREZ, G. I. \& VALENZUELA, R. O. Alteraciones de número en dentición de pacientes entre 2 y 12 años de edad con disrafias labio alvéolo palatina atendidos en la Unidad de Odontopediatria del Hospital Regional Antofagasta, Chile. Int. J. Odontostomat., 8(3):481-490, 2014.

Tabla VIII. Distribución de pacientes de 2 a 6 años según anomalías de número y cantidad de dientes afectados.

\begin{tabular}{ccccc}
\hline \multirow{2}{*}{ Número de dientes } & \multicolumn{4}{c}{ Alteración de número } \\
& \multicolumn{2}{c}{ Agenesia } & \multicolumn{1}{c}{ Supernumerario } \\
\cline { 2 - 5 } & $\mathrm{n}$ & $\%$ & $\mathrm{n}$ & $\%$ \\
\hline 1 & 19 & 67,8 & 10 & 90,9 \\
2 & 8 & 28,6 & 1 & 9,1 \\
3 o mas & 1 & 3,6 & --- & 0 \\
Total & 28 & 100 & 11 & 100 \\
\hline
\end{tabular}

nían dientes supernumerarios presentaron 1 diente afectado, el 16,7\% mostraron 2 dientes afectados (Tabla IX).

Tabla IX. Distribución de niños de 7 a 12 años según anomalías de número y cantidad de dientes afectados.

\begin{tabular}{ccccc}
\hline \multirow{2}{*}{ Número de dientes } & \multicolumn{4}{c}{ Alteración de número } \\
& \multicolumn{2}{c}{ Agenesia } & \multicolumn{2}{c}{ Supernumerario } \\
\cline { 2 - 5 } & $\mathrm{n}$ & $\%$ & $\mathrm{n}$ & $\%$ \\
\hline 1 & 8 & 61,5 & 5 & 83,3 \\
2 & 4 & 30,8 & 1 & 16,7 \\
3 o mas & 1 & 7,7 & --- & 0 \\
Total & 13 & 100 & 6 & 100 \\
\hline
\end{tabular}

En relación al diente más afectado en los niños con agenesia según edad, se pudo determinar que el $78,6 \%$ entre 2 a 6 años tenía afectado el incisivo lateral y el $21,4 \%$ el incisivo central, sin embargo la totalidad de los niños entre 7 y 12 años presentaron afectado el incisivo lateral. En ninguno de los dos grupos de edad se presentó agenesia del canino (Tabla X).

Las alteraciones de número y lado donde se

Tabla X. Distribución de los niños con agenesia dentaria según morfología del diente afectado y edad.

\begin{tabular}{lcccc}
\hline \multirow{2}{*}{\multicolumn{1}{c}{ Diente }} & \multicolumn{4}{c}{ Agenesia } \\
& \multicolumn{2}{c}{$2-6$ años } & \multicolumn{2}{c}{$7-12$ años } \\
\cline { 2 - 5 } & $\mathrm{n}$ & $\%$ & $\mathrm{n}$ & $\%$ \\
\hline Incisivo Central & 6 & 21,4 & 0 & 0 \\
Incisivo Lateral & 22 & 78,6 & 13 & 100 \\
Canino & 0 & 0 & 0 & 0 \\
Total & 28 & 100 & 13 & 100 \\
\hline
\end{tabular}

ubica la fisura en niños de 2 a 6 , se observó que la agenesia se ubica en el $89,3 \%$ al lado de la fisura y en un $10,7 \%$ en el lado opuesto, Los supernumerarios se ubicaron de igual forma en mayor frecuencia en el lado de la fisura en el $90,9 \%$ de los casos, solo un $9,1 \%$ se ubica en el lado opuesto (Tabla XI).
Tabla XI. Distribución de los niños de 2 a 6 años según anomalías de número y lado donde se ubica la fisura.

\begin{tabular}{lcccc}
\hline Ubicación en relación a la & \multicolumn{4}{c}{ Alteración de número } \\
Fisura & Agenesia & \multicolumn{2}{c}{ Supernumerario } \\
\cline { 2 - 5 } & $\mathrm{n}$ & $\%$ & $\mathrm{n}$ & $\%$ \\
\hline Lado de la Fisura & 25 & 89,3 & 10 & 90,9 \\
Lado Contrafisura & 3 & 10,7 & 1 & 9,1 \\
Total & 28 & 100 & 11 & 100 \\
\hline
\end{tabular}

Según anomalías de número y el lado donde se ubica la fisura, la distribución en niños de 7 a 12 años, se pudo observar que el $53,8 \%$ de la agenesia se presentó en el lado de la fisura y el $46,2 \%$ en el lado opuesto. Los supernumerarios por su parte se presentaron con igual frecuencia en el lado de la fisura y en el lado opuesto de la misma con el 50\% (Tabla XII).

Tabla XII. Distribución de los niños de 7 a 12 años según anomalías de número y lado donde se ubica la fisura.

\begin{tabular}{lcccc}
\hline \multirow{2}{*}{$\begin{array}{l}\text { Ubicación en relación a la } \\
\text { Fisura }\end{array}$} & \multicolumn{4}{c}{ Alteración de número } \\
\cline { 2 - 5 } & $\mathrm{n}$ & $\%$ & $\mathrm{n}$ & $\%$ \\
\hline Lado de la Fisura & 7 & 53,8 & 3 & 50 \\
Lado Contrafisura & 6 & 46,2 & 3 & 50 \\
Total & 13 & 100 & 6 & 100 \\
\hline
\end{tabular}

\section{DISCUSIÓN}

Las alteraciones de número en la dentición de pacientes portadores de Fisura Labio Palatina se explican porque éstas se producen durante el proceso de desarrollo de cara y paladar, eventos de alta complejidad donde cualquier interrupción o modificación en el patrón de desarrollo y crecimiento prenatal del individuo dará origen a malformaciones congénitas (Palominos \& Montenegro, 2008).

Las FLAP se produce entre la cuarta y sexta semana del desarrollo embrionario, se encuentra entre las malformaciones más frecuentes que se presentan en la especie humana, aunque con distinta incidencia entre diferentes poblaciones como lo comprueban múltiples estudios epidemiológicos. Los factores etiológicos que se relacionan con las FLAP son múltiples, existiendo una interacción entre factores genéticos y ambientales como la influencia de teratógenos o fuerzas mecánicas que afectan al individuo en las primeras etapas del desarrollo; y que favorecerán la aparición de estas alteraciones (Merrit, 
GUTIÉRREZ, G. I. \& VALENZUELA, R. O. Alteraciones de número en dentición de pacientes entre 2 y 12 años de edad con disrafias labio alvéolo palatina atendidos en la Unidad de Odontopediatria del Hospital Regional Antofagasta, Chile. Int. J. Odontostomat., 8(3):481-490, 2014.

2005). Así como también el consumo de fármacos en el primer trimestre del embarazo, alcoholismo y drogadicción de los padres, tendrán gran relevancia en la aparición de estas anomalías como la fisura velopalatina.

Según Ford et al. (2010) hay muchas clasificaciones de las fisuras de acuerdo a la enorme variabilidad que pueden presentar las fisuras labiopalatinas, muchos autores a través del tiempo han propuesto diferentes sistemas de clasificación, fundamentados en criterios embriológicos, anatómicos, odontológicos, quirúrgicos, etc. Se destacan la clasificación de Davis \& Ritchie (Rossel, 2006), que señala tres grupos: I Fisura de labio, II Fisura de paladar y III Fisura de labio y paladar. Kernahan \& Stark en el año 1958 (Corbo \& Marimon, 2001), presentaron su clasificación de labio y paladar hendidos, introduciendo conceptos de paladar primario y paladar secundario, se consiguió integrar dichos conceptos, correlacionarlos con el proceso embriopatogénico, consideran dos grupos básicos: Fisura del paladar primario (labio y premaxila) y Fisura del paladar secundario (paladar duro y blando posterior al foramen incisivo).

Embriológicamente se clasifican en cuatro grupos: Fisuras pre-palatinas o de paladar primario (que puede afectar el labio con o sin compromiso del alvéolo), Fisuras de paladar secundario (que pueden comprometer el paladar óseo o blando), mixtas (con compromiso del labio y paladar) y Fisuras raras de menor ocurrencia.

Según la clasificación de Olin, estas pueden ser i) Fisura Labial: defecto congénito en el labio superior uni o bilateral, donde falla la fusión de la prominencia maxilar con la prominencia nasal media. Se piensa que puede ser causado por la falta de migración del mesodermo en la región cefálica. ii) Fisura Palatina: fisura congénita del paladar blando y/o duro, debido a la falta de fusión. Se produce cuando el paladar no se cierra completamente, dejando una abertura que puede extenderse dentro de la cavidad nasal. La hendidura puede afectar a cualquier lado del paladar. Puede extenderse desde la parte frontal de la boca (paladar duro) hasta la garganta (paladar blando); y iii) Fisura labio palatina Uni o bilateral: fisura congénita de labio y paladar duro y/o blando, debido a la falta de fusión. Constituyen deficiencias estructurales congénitas debidas a la falta de coalescencia entre algunos de los procesos faciales embrionarios en formación. Se manifiestan, por tanto, precozmente en la vida intrauterina, más específicamente en el período em- brionario e inicio del período fetal.

Es importante mencionar que Wu et al. (2011) compararon las anomalías dentales asociadas con cada tipo de fisura. La frecuencia $(20 \%)$ de anomalías dentales en el área incisiva maxilar del grupo fisura palatina (FP) fue significativamente menor que en los otros grupos. La frecuencia de agenesia de incisivos laterales maxilares (ILM) aumentaba con la severidad de la fisura. Los dientes supernumerarios y los incisivos inferiores mostraron la tendencia opuesta. Los patrones de distribución de agenesia y en grano de arroz de ILM en varones fueron consistentes para los tres tipos de fisuras unilaterales; no así en las mujeres. Al considerar las características de las anomalías dentales entre las tres fisuras unilaterales, se encontró que las agenesias de ILS, los dientes supernumerarios y los incisivos inferiores estaban relacionados con la severidad de la fisura. EI ILM fue el diente más afectado en el área de la fisura. La frecuencia de agenesia de ILM y laterales en grano de arroz no presentaron dismorfismo sexual, pero el patrón de distribución fue diferente dependiendo del sexo.

Por otro lado, Tannure et al. (2011) evaluaron mediante un estudio de meta análisis si los individuos que nacen con fisuras no sindrómicas orales muestran una mayor frecuencia de anomalías dentales, resultando una asociación significativa entre la agenesia dental y fisuras orales. En los análisis posteriores, se mostró una asociación positiva entre supernumerarios y anomalías morfológicas de la corona en las fisuras orales. Concluyeron que la evidencia sugiere que hay un mayor número de anomalías dentales en la dentición permanente en los individuos que nacen con fisuras orales. En el mismo año, de Lima Pedro et al. (2011), en un estudio de casos y controles determinaron la presencia de patrones específicos de defectos dentales en un grupo de individuos nacidos con fisuras y encontraron que los individuos que nacen con fisura presentan anomalías dentales mucho más en comparación con los controles $(p=0,0001)$. La más frecuente fue la agenesia dental $(n=53, p=0,001)$, seguido de los dientes supernumerarios $(n=14, p=0,11)$ y malposición dentaria $(n=11, p=0,33)$. En cuanto a las agenesias, los incisivos laterales maxilares estaban más frecuentemente ausentes en el grupo de los pacientes con fisuras (31/86), mientras que los individuos de control presentaron más agenesia de los segundos premolares mandibulares (19/45). Las anomalías de número son las más asociadas a las fisuras labio alvéolo palatinas.

En la investigación de Fernández Sánchez \& 
GUTIÉRREZ, G. I. \& VALENZUELA, R. O. Alteraciones de número en dentición de pacientes entre 2 y 12 años de edad con disrafias labio alvéolo palatina atendidos en la Unidad de Odontopediatria del Hospital Regional Antofagasta, Chile. Int. J. Odontostomat., 8(3):481-490, 2014.

Magán Moya (2010), se plantea que los varones son normalmente los más afectados, excepto en el caso de la fisura aislada del paladar, que presentando una frecuencia equivalente a uno de cada 3.000 nacimientos, tiene mayor incidencia en el sexo femenino. Los estudios de Corbo \& Marimon, también comprobaron que las fisuras de labios son más frecuentes en los varones, mientras que las fisuras aisladas del paladar son más comunes en las mujeres.

Con relación a los tipos de fisura, el más frecuentemente encontrado fue la fisura labiopalatina unilateral con un $64,79 \%$ del total de las fisuras, en concordancia con los estudios de Shapira et al. (1999) y Lekkas et al. (2000); y una mayor tendencia al sexo masculino al igual que lo encontrado por Lekkas et al., Alas et al. (2007) y Baek \& Kim (2007).

De los pacientes que presentaron agenesias estos se encontraron con mayor frecuencias en las FLAP unilateral con un $57,75 \%$, en contraste con lo que encontró Shapira et al. (2000), que se presentaban con mayor frecuencia en los pacientes con FLAP bilateral considerando la severidad, esta diferencia puede explicarse porque en las fisuras unilaterales también las hay de diferente severidad, siendo algunas muy amplias que ocasionan alteraciones de número.

La frecuencia de agenesias en el lado de la fisura fue de un $89,3 \%$, resultado similar a lo encontrado por Baek \& Kim, esto se explica por la ausencia del germen en la zona afectada por la fisura.

El diente más afectado en las agenesias fue el Incisivo lateral con un $78,6 \%$ de los niños entre 2 a 6 años y en un $100 \%$ en los niños entre 7 y 12 años, resultados que son comparables a lo descrito por la mayoría de estudios. Cabe destacar que no se presentaron agenesia de caninos.

En relación a la agenesia del incisivo lateral, autores como Shapira et al. (2000), señalan en estudios realizados que el diente que generalmente se encuentra ausente en los pacientes con labio y paladar hendido son los incisivos laterales maxilares permanentes; resultados similares y consistentes con los encontrados en este estudio. Por otro lado, Lourenço Ribeiro et al. (2003) reportan que generalmente el incisivo lateral maxilar, es el diente que se encuentra como supernumerario, y este se localiza distal de la hendidura del paladar. Smahel et al. (1996), difieren de los resultados observados en este estudio, pues exponen que los dientes que generalmente se encuen- tran ausentes son los incisivos centrales maxilares, esto es cuando los pacientes presentan un labio y paladar hendido unilateral. Además Aizenbud et al. (2005) también mencionan en su investigación que generalmente los dientes que se encuentran ausentes en los pacientes con labio y paladar hendido son los incisivos laterales, resultados similares a los de esta investigación.

La frecuencia de agenesia dentaria en el lado de fisura que se muestra en los resultados de este estudio, están en concordancia con las mostradas por Baek \& Kim y Shapira et al. (2000), autores que reportan mayor frecuencia de agenesia en el lado de fisura.

Respecto a la cantidad de dientes afectados Se pudo observar que el $61,5 \%$ de los niños con agenesia tenían un diente afectado, el 30,8\% mostraron 2 dientes afectados y solo el $7,7 \%$ presentó 3 o más dientes afectados, similar a los resultados mostrados por Heliövaara et al. (2004).

En relación a la frecuencia de dientes supernumerarios se obtuvo un $23,94 \%$ similar a los resultados obtenidos por Alas et al. con un 27,6\% y Slayton et al. (2003) con un $27 \%$.

Un niño tendrá un riesgo de un 3 a un $5 \%$ de presentar FLAP, si uno de sus padres la presenta; este riesgo aumentará a un $40 \%$, si además uno de sus hermanos nació con fisura.

Es importante colocar atención en las cifras de los datos epidemiológicos de FLAP; la tasa mundial es de 1 por cada 1200 nacidos vivos; en Chile de 1,8 por 1000 nacidos vivos y en la Región de Antofagasta de 1 por 259 nacidos vivos. Debemos considerar que nuestra región está constantemente expuesta a contaminación ambiental derivada especialmente de la extracción o traslado de minerales, los que originan alta contaminación como polvo en suspensión o a la presencia de metales pesados en el ambiente; éstos podrían considerarse como posibles agentes teratógenos asociados a estas malformaciones. La exposición a contaminantes está asociada además a alteraciones del desarrollo cognitivo de los individuos cuando éstos han sido expuestos durante el período pre y post-natal (Dietrich et al., 1993).

Los datos epidemiológicos en relación a las FLAP y su alta incidencia de éstas en la Región de Antofagasta, nos obligan a estar atentos frente a la aparición de estas malformaciones y a los factores 
GUTIÉRREZ, G. I. \& VALENZUELA, R. O. Alteraciones de número en dentición de pacientes entre 2 y 12 años de edad con disrafias labio alvéolo palatina atendidos en la Unidad de Odontopediatria del Hospital Regional Antofagasta, Chile. Int. J. Odontostomat., 8(3):481-490, 2014.

de riesgos a los que está expuesta la población como por ejemplo la situación medioambiental de nuestra
Región.

REFERENCIAS BIBLIOGRAFICAS

GUTIÉRREZ, G. I. \& VALENZUELA, R. O. Dentition number alterations in patients between 2 and 12 years of age with cleft alveolus palate examined at the pediatric dental unit of the Regional Hospital Antofagasta, Chile. Int. J. Odontostomat., 8(3):81-490, 2014.

ABSTRACT: The present study was carried out to research alterations of teeth in 71 patients, whose ages were between two and twelve years old and who exhibited alveolus cleft lip palate. These patients were treated at The Regional Hospital in Antofagasta around April 2004 and July 2010, therefore, we used the tabs clinics, panoramic radiograph, occlusal and periapical radiographs. A prevalence of tooth agenesis in $57.75 \%$ of supernumerary teeth in $23.95 \%$ was found and $18.4 \%$ of children did not show any modification of number. In connection with the distribution by sex of the cleft lip and cleft lip bilateral alveolar palatine presented a high frequency in boys, and the palate clefts with fissure unilateral alveolus cleft lip were frequently observed in girls. Regarding to the types of cleft lip, the most frequent found was the alveolar cleft lip palate unilateral with $64.70 \%$ over the total of the fissures. On the other hand, the frequency of agenesis, related to fissure was $89.3 \%$. The most affected tooth in agenesis was the lateral incisor with $78.6 \%$ in children whose ages were from 2 to 6 years old, and $100 \%$ in children from 7 to 12 .It was also observed that $61.5 \%$ of children with agenesis had one affected tooth, 30.8 $\%$ had two affected teeth and only $7.7 \%$ had three or more affected teeth.

KEY WORDS: fissure, tooth agenesis, supernumerary teeth.

Aizenbud, D.; Camasuvi, S.; Peled, M. \& Brin, I. Congenitally missing teeth in the Israeli cleft population. Cleft Palate Craniofac. J., 42(3):3147, 2005.

Alas, C. I. L.; Gurrola Martínez, B.; Díaz Cepeda, L. F. \& Casasa Araujo, A. Incidencia de dientes ausentes y supernumerarios en pacientes con labio y paladar hendido. Rev. Latinoam. Ortod. Ortop. [online] 2007. Disponible en: https:// www.ortodoncia.ws/publicaciones/2007/art8.asp

Baek, S. H. \& Kim, N. Y. Congenital missing permanent teeth in Korean unilateral cleft lip and alveolus and unilateral cleft lip and palate patients. Angle Orthod., 77(1):88-93, 2007.

Corbo, M. T. \& Marimón, M. E. Labio y paladar fisurados. Aspectos generales que se deben conocer en la atención primaria de salud. Rev. Cubana Med. Gen. Integr., 17(4):379-85, 2001.

de Lima Pedro, R.; Faría, M. D.; de Castro Costa, M. \& Vieira, A. R. Dental anomalies in children born with clefts: a case-control study. Cleft Palate Craniofac. J., 49(6):e64-8, 2012.

Dietrich, K. N.; Berger, O. G.; Succop, P. A.; Hammond, P. B. \& Bornschein, R. L. The developmental consequences of low to moderate prenatal and postnatal lead exposure: intellectual attainment in the Cincinnati Lead Study Cohort following school entry. Neurotoxicol. Teratol., 15(1):37-44, 1993.

Fernández Sánchez, J. \& Magán Moya, A. El paradigma estético y funcional del paciente con fisura labiopalatina. Ortod. Esp., 50(2):382-97, 2010.

Ford M., A.; Tastets H., M. E. \& Cáceres R., A. Tratamiento de la fisura labio palatina. Rev. Med. Clin. Condes., 21(1):16-25, 2010.

Helióvaara, A.; Ranta, R. \& Rautio, J. Dental abnormalities in permanent dentition in children with submucous cleft palate. Acta Odontol. Scand., 62(3):129-31, 2004.

Lekkas, C.; Latief, B. S.; ter Rahe, S. P. \& KuijpersJagtman, A. M. The adult unoperated cleft patient: absence of maxillary teeth outside the cleft area. Cleft Palate Craniofac. J., 37(1):1720, 2000.

Lourenço Ribeiro, L.; Teixeira Das Neves, L.; Costa, B. \& Ribeiro Gomide, M. Dental anomalies of the permanent lateral incisors and prevalence of hypodontia outside the cleft area in complete unilateral cleft lip and palate. Cleft Palate Craniofac. J., 40(2):172-5, 2003.

Merritt, L., Part 1. Understanding the embryology and 
GUTIÉRREZ, G. I. \& VALENZUELA, R. O. Alteraciones de número en dentición de pacientes entre 2 y 12 años de edad con disrafias labio alvéolo palatina atendidos en la Unidad de Odontopediatria del Hospital Regional Antofagasta, Chile. Int. J. Odontostomat., 8(3):481-490, 2014

genetics of cleft lip and palate. Adv. Neonatal Care., 5(2):64-71, 2005.

Ministerio de Salud (MINSAL). Guía Clínica Fisura Labiopalatina. Santiago, Ministerio de Salud (MINSAL), 2009.

Palominos, H. \& Montenegro, M. Tratamiento interdisciplinario de las fisuras labio palatinas. Santiago, Monasterio Ed., 2008.

Rossell, P. P. Nueva clasificación de severidad de Fisuras Labiopalatinas del Programa Outreach Surgical Center Lima - Perú. Acta Méd. Peruana, 23(2):59-66, 2006.

Shapira, Y.; Lubit, E. \& Kuftinec, M. M. Congenitally missing second premolars in cleft lip and cleft palate children. Am. J. Orthod. Dentofacial Orthop., 115(4):396-400, 1999.

Shapira, Y.; Lubit, E. \& Kuftinec, M. M. Hypodontia in children with various types of clefts. Angle Orthod., 70(1):16-21, 2000.

Slayton, R. L.; Williams, L.; Murray, J. C.; Wheeler, J. J.; Lidral, A. C. \& Nishimura, C. J. Genetic association studies of cleft lip and/or palate with hypodontia outside the cleft region. Cleft Palate Craniofac. J., 40(3):274-9, 2003.

Smahel, Z.; Tomanová, M. \& Müllerová, Z. Position of upper permanent central incisors prior to eruption in unilateral cleft lip and palate. Cleft Palate Craniofac. J., 33(3):219-24, 1996.

Tannure, P. N.; Oliveira, C. A.; Maia, L. C.; Vieira, A. R.; Granjeiro, J. M. \& de Castro Costa, M. Prevalence of Dental Anomalies in Nonsyndromic Individuals With Cleft Lip and Palate: A Systematic Review and Meta-analysis. Cleft Palate Craniofac. J., 49(2):194-200, 2011.

Wu, T. T.; Chen, P. K.; Lo, L. J.; Cheng, M. C. \& Ko, E. W. The characteristics and distribution of dental anomalies in patients with cleft. Chang Gung Med. J., 34(3):306-14, 2011.

Dirección para correspondencia :
Dra. Irene Gutiérrez Guerra

Servicio Dental

Hospital Regional de Antofagasta

Avda. Argentina 1962, subterráneo

Antofagasta

CHILE

Email : irenegutierrezguerra@hotmail.com

Recibido : 08-09-2014

Aceptado: 18-12-2014 